\title{
Molecular and Conventional Identification of Malassezia Species in Patients with Pityriasis Versicolor
}

\author{
Randa EL-Sherbini ${ }^{1}$, Noha Elmashad ${ }^{2}$, Hanan Fathy ${ }^{3}$, \\ Mohammed Elshaer ${ }^{2 *}$ and Salah Agha $^{2}$ \\ ${ }^{1}$ Mansoura Ophthalmology Hospital, Egypt \\ ${ }^{2}$ Clinical Pathology Department, Mansoura Faculty of Medicine, Egypt \\ ${ }^{3}$ Dermatology Department, Mansoura Faculty of Medicine, Egypt \\ *Corresponding author
}

\section{A B S T R A C T}

\begin{tabular}{|l|}
\hline Ke y w o r d s \\
Malassezia, \\
Pityriasis \\
versicolor, \\
Restriction \\
Fragment Length \\
Polymorphism \\
\hline Article Info \\
\hline $\begin{array}{l}\text { Accepted: } \\
\text { 12 May 2020 } \\
\text { Available Online: } \\
\text { 10 June } 2020\end{array}$ \\
\hline
\end{tabular}

Pityriasis versicolor (PV) is a common health problem caused by genus Malassezia. Identification of Malassezia spp. has been carried out mostly through morphological and biochemical analyses. Various molecular biological techniques are being preferred as they are species-specific, more accurate and less timeconsuming. The purpose of this study was to identify Malassezia species on the skin of patients with pityriasis versicolor and healthy individuals. Also, antifungal susceptibility testing was performed on the isolated Malassezia species. A case-control study including 100 individuals; 50 clinically suspected pityriasis versicolor patients attending Mansoura University Hospitals, Egypt and 50 healthy control individuals, was carried out. Characterization of Malassezia species was performed phenotypically by conventional, culture-based methods, biochemical tests, and automated system. In addition, genomic DNA was extracted from isolated colonies for PCR amplification of the highly conserved 26S rDNA region with further species level identification by Restriction Fragment Length Polymorphism using Hha1 and FOK1 enzymes. The association of Malassezia species with epidemiological profile and clinical characteristics were evaluated. A $84 \%$ of PV samples and $10 \%$ of control samples were positive by potassium hydroxide $(\mathrm{KOH})$ while $78 \%$ of PV samples and $18 \%$ of control samples yielded growth in culture with statistical differences $(\mathrm{p}<0.001$, for both methods). By phenotypic methods, isolates from patients were identified as: M. furfur (87.2\%), and M. globosa, (7.7\%), while in healthy controls: M. furfur (77.8\%), and M. globosa, (11.1\%). ByPCR-RFLP technique, M. furfur (89.7\%), and M. globosa $(10.3 \%)$, while in healthy controls: M. furfur $(88.9 \%)$, and M. globosa, $(11.1 \%)$. The highest proportion of sensitivity of Malassezia was detected to flucytosine, micafungin, caspofungin, voriconazole (100\%) in both cases and control. Malassezia furfur and Malassezia globosa are the commonly encountered species in both PV patients and healthy human skin. PCR-RFLP method is considerably accurate technique in the identification of Malassezia species.

\section{Introduction}

Yeasts of the genus Malassezia which consists of 17 species, are lipophilic yeasts, which are a part of the normal skin flora(1). Malassezia yeasts inhabit various body sites including scalp, forehead, shoulder, abdomen, lower axilla, groin and forearm, due to an increased thickness of sebaceous organs at to be these sites (2). It is associated with numerous superficial skin diseases as pityriasis versicolor, seborrheic dermatitis, folliculitis, and atopic dermatitis (3).

Pityriasis (tinea) versicolor is a mild, chronic infection of the skin caused by Malasseziayeasts, and characterized by discrete or confluent, scaly oval to round 
macules scattered over characteristic areas of the body, including the upper trunk, neck, and upper arms. Generally, patients with tinea versicolor are asymptomatic, although minority of some patients complain of mild pruritus(4). Different Malassezia species have shown various antifungal susceptibility patterns. Therefore, it is quite important to identify the Malassezia species in order to choose the most sensitive antifungal drug (1). The diagnostic methods used to confirm the presence of Malassezia yeasts include direct microcopy, culture based methods (often a combination of morphological features of the isolate combined with biochemical tests), molecular based methods such as Polymerase Chain Reaction techniques, and Matrix Assisted Laser Desorption/Ionization-Time Of Flight mass spectrometry(5)

The aim of this work was to identify Malassezia species on the skin of patients with pityriasis versicolor and healthy individuals. Also, antifungal susceptibility testing was performed on the isolated Malassezia species.

\section{Ethical consideration}

Informed consents were obtained from all patients and healthy subjects. The study protocol was approved by the local Ethics Committee of Mansoura University, Egypt.

\section{Study locality}

The study was conducted at outpatient clinics of Dermatology and Clinical Pathology departments of Mansoura University Hospitals.

\section{Inclusion criteria}

A total of 50 clinically suspected pityriasis versicolor patients (32 male and 18 females with a mean age of 32.1 years) were included. Their characteristic lesions were scaly hypopigmented or hyperpigmented welldefined macules, with slight desquamation and color ranging from white to brown. Fifty healthy individuals of matched age and sex (33 male and 17 females with a mean ageof 31.7 years) were included as a control group.

\section{Exclusion criteria}

Patients taking topical or oral antifungal agents in the previous two months, patients treated with immunosuppressive drugs, and patients with other skin diseases caused by Malassezia.

\section{Sample collection}

Scales with repeated scraping were collected from lesions in patients and from healthy controls. They were obtained from chest, back, and neck. Skin scrapings were done by a sterile surgical blade after use of cotton soaked with alcohol 70\%. The scales were collected in clean dark colored paper squares.

\section{Materials and Methods}

\section{Direct microscopy}

Scales specimens were subjected to direct examination by placing on a clean slide mounted with a drop of $20 \% \mathrm{KOH}$ and covered with a cover slip. The slide was examined under a microscope (40X). The presence of characteristic spaghetti and meatballs appearance (shortly curved hyphae and round yeasts) confirmed the presence of Malassezia.

\section{Culture}

The samples were inoculated on two Sabouraud's Dextrose Agar (SDA) slants supplemented with cycloheximide and chloramphenicol. One slant was overlaid with olive oil and the other without for isolation of non-lipid-dependent $M$. pachydermatis. 
Inoculated slants were incubated at $37^{\circ} \mathrm{C}$ for 1-2 weeks and examined at frequent intervals.

Smears from the colonies were stained with Gram's stain and examined under microscope for identification of Malassezia. After two weeks of incubation, the culture slants without growth were considered negative and discarded. In order to achieve pure cultures, for each positive sample colonies were grown in tubes containing $5 \mathrm{ml}$ of Sabouraud's dextrose agar in slants. They were overlaid with glycerol as a cryoprotective agent at concentrations of $10 \%$, covered with parafilm and stocked at $-80^{\circ} \mathrm{C}$. Fresh subculture was prepared from frozen samples for subsequent methodology.

\section{Identification of the isolates}

Identification of Malassezia isolates was done by biochemical tests, automated identification by Vitek2 and Matrix-assisted laser desorption ionization time-of-flight mass spectrometry (MALDI-TOF, Vitek MS, Biomérieux, France) for confirmation of identification pattern obtained by Vitek 2 .

Antifungal susceptibility testing by Vitek 2 system

Molecular identification of Malassezia species using 26S rDNA PCR-restriction fragment polymorphism (RFLP).

DNA Extraction by QIA amp DNA mini kit (Qiagen, Germany) according to manufacturer instructions.

PCR amplification (Thermal cycler; Biometra, Germany): two primers: forward, 5-TAACAAGGATTCCCCTAGTA-3 and reverse, 5- ATTACGCCAGCATCCTAAG-3 were used to amplify the internal transcribed spacer (ITS) region in the rDNA gene in all Malassezia spp. PCR Master Mix was prepared in a final volume of $25 \mu$ laccording to Emerald Amp GT PCR master mix (Takara Bio, USA).

Fragmentation of PCR products by restriction enzymes: The enzymes Fast Digest FOK1, and HhaI (Thermofisher, Germany)were selected according to Mirhendi et al., (2005)(6).

\section{Statistical analysis}

The collected data was revised, coded, tabulated and introduced to a PC using Statistical package for Social Science (IBM SPSS Statistics for Windows, Version 25.0. Armonk, NY: IBM Corp).

\section{Results and Discussion}

A total of 50 clinically suspected PV patients (32 male and 18 females with a mean age of 32.1 years) were included. Pityriasis versicolor was more prevalent in patients with mean age of 32.1 years and more in males $(64 \%)$, than in females $(36 \%)$ (Table 1).

A significantly higher direct $\mathrm{KOH}$ results among patients than healthy individuals (84\% vs $10 \%$ ). Also, a significantly higher isolation rate of Malasseziacolonies was yielded among PV patients than healthy individuals $(78 \%$ vs $18 \%$ ). The colonies of Malasseziawas creamy, moist, pasty colonies on the SDA slants(Figure 1).Gram stained smears from the colonies were positive for budding yeast cells.

By automated methods, 9 Malassezia isolates from healthy individuals were identified as $M$. furfur $(77.8 \%)$ and $M$. globosa $(11.1 \%)$ that were established by PCR as $M$. furfur $(88.9 \%)$ and $M$. globosa $(11.1 \%)$ whereas out of 50 clinically suspected patients, 39samples were confirmed by isolation of Malassezia isolates. Phenotypically, isolates of Malassezia 
revealed two species: $M$. furfur34 (87.2\%), and $M$. globosa 3 (7.7\%), while $2(5.1 \%)$ were unidentified isolates. By using PCR technique, all isolates (100\%) were identified (Table 2-3).

PCR method produced single PCR product of approximately $580 \mathrm{bp}$ that was further studied by RFLP analysis using Hha1 and FOK1 restriction enzymes. Band analysis by restriction enzymes show Malassezia furfur (two bands at 311 and $429 \mathrm{bp}$ ), Malassezia globosa (two bands at 306 and $361 \mathrm{bp}$ ). The tested 50Malassezia isolates from patients revealed two species: M. furfur 35 (89.7\%), and M. globose 4 (10.3\%), with higher identification rate of isolates by PCR-RFLP compared to phenotypic methods $(\mathrm{p}=0.940)$ (Figure2).

The isolated Malassezia furfur and globosa were sensitive to flucytosine, micafungin, caspofungin, voriconazole (100\%) in both cases and control (Table 4).

Pityriasis versicolor (PV) is a mild, chronic infection of the skin caused by Malassezia yeasts, characterized by discrete or confluent, scaly, dark or depigmented patches, mainly on the upper trunk but this can extend to the neck, abdomen and other sites, although the peripheries are usually spared. Pityriasis versicolor occurs in both tropical, where it may be very common, and temperate climates and affects both genders (5).

Male represents 64\% in patients' group (Table 1). The role of sex in development of pityriasis versicolor is unclear. Some studies found that $\mathrm{PV}$ is more common in males than females (7-10).

The higher incidence of PV in males may be due to their outdoor activities(11). Other studies show that the incidence may be higher in females because of beauty and skin hygiene products(12).PV was more frequent in the age of 20-40 years (Table 1). Similarly, Crespo et al., (2000); Gupta et al., (2002); and Moniri et al., (2009) reported that the disease peaksat this age $(13-14,10)$.

In this study, direct microscopy using $\mathrm{KOH}$ $20 \%$ in PV cases was positive in $84 \%$ whereas in healthy skin yeast and filaments were present in $10 \%$ of samples. Similar results were reported by Shah et al., (2013) and Shoeib et al., (2013) (9-15).

Culture on Sabouraud's dextrose agar(SDA) with olive oil was positive in $78 \%$ of patients and $18 \%$ of healthy persons (Table 2). Archana et al., (2015)showed positive rate of 70\%, while Shah et al., (2013), and Jang et al., (2009) showed (50\%) and(62\%) respectively(16, 9, 17). On the other hand, higher isolation rate was reported in India(96.66\%)by Chaudhary et al., (2010), and in Iran (88.4\%) by Shokoshi et al., (2009)(1819).

All isolates obtained from culture showed gram positive budding yeasts with urease and catalase positive results. Identification of isolates and antifungal susceptibility testing were carried out by Vitek 2 system which gave Malassezia furfur in (30/39) of patients and in (7/9) of healthy control. Malassezia globosa could not be identified by vitek 2 as it is not present in its identification database.

Furthermore, MALDI-TOF was used for confirmation of identification pattern obtained by Vitek 2.It revealed Malassezia furfur in (34/39) of patients, and in (7/9) of control subjects, and Malassezia globosa in (3/39) of patients, and in (1/9) of healthy persons. Three isolates were unidentified by MALDITOF. The low number of strains included in the database may lead to non-identifiable results (20).

The 26S $r D N A$, which was targeted by PCRRFLP in this study, contains highly conserved 
base sequences and enough sequence variation that can serve as markers for identification of Malassezia species. It requires only two restriction enzymes, Hha 1 , and FOKI, and has been proven to be technically easier than other molecular techniques (21).PCR- RFLP identified Malassezia furfur in $89.7 \%$ (35/39) of patients and in $88.9 \%(8 / 9)$ of healthy subjects and in $10.3 \%$ (4/39) of patients and $11.1 \%$ (1/9) of healthy subjects.

MALDI-TOF was in concordance with PCR RFLP results for identification of the two Malassezia spp. except one isolate which was identified as $M$. furfur by MALDI-TOF but identified as $M$. globosa by RFLP. This discordance may be due to the presence of multiple species in a single culture (cocolonization) than true misidentification.

Identification by using MALDI-TOF revealed that Malassezia furfur was more frequent than $M$. globosa $(89.7 \%$ and $10.3 \%$, respectively). In healthy control, they were $88.9 \%$ and $11.1 \%$, respectively. Similarly, Sharma et al., (2016) showed that Malassezia furfur was the most predominant isolate in patients with PV (77.3\%) followed by $M$. globosa in $12.4 \%$. Also, in a study by Li et al., (2020), Malassezia furfur and Malassezia globosa were present in $67.86 \%$ and $18.88 \%$ of patients (22-23).

On the other hand, Gupta et al., (2004), showed that $M$. symbodialis was the main isolate in PV patients and M. globosa as the predominant isolate in tropical regions (24). Malassezia sympodialis was the dominant species in healthy controls in studies by Falk et al., (2005) and Gupta et al., (2001)(25-26). Other studies have reported $M$. globosa was the main isolate in healthy individuals followed by $M$. sympodialis (12). Detection of different ratios of Malassezia species in patients with PV patients in different parts of the world could be due to the use of different culture media, different sampling methods (13), climatic regions and characteristics of patients (24).

The predominance of $M$. furfur in this study may be due to higher temperature and humidity that may play a role in its pathogenicity. A gene encoding a secreted lipase of $M$. furfur possibly associated with both its growth, and pathogenicity was cloned and characterized, and it was found that this gene is most active at temperature more than $40^{\circ} \mathrm{C}(27)$. M. furfur also produces an indole alkaloid pityriacitrin which can protect this fungus against ultraviolet exposure and renders $M$. furfur more resistant to sun exposure(28).

All Malassezia isolates were sensitive to flucytosine, micafungin, caspofungin, voriconazole $(100 \%)$ in both cases and control. The results of other in vitro susceptibility studies have shown variations in the susceptibility of Malassezia spp. to various antifungal agents. In 2014, Rojas et $a l$., showed that fluconazole is active against M. sympodialis and $M$. slooffiae, but with little or no activity against Malassezia globosa and Malassezia restricta(29). Hammer et al., (2000) found that ketoconazole in more active against $M$. furfur strains isolated from systemic infection than econazole and miconazole. Discrepancy of results from other studies might be due to lack of a standardized protocol for Malassezia susceptibility testing (30). 
Table.1 Demographic data among studied groups

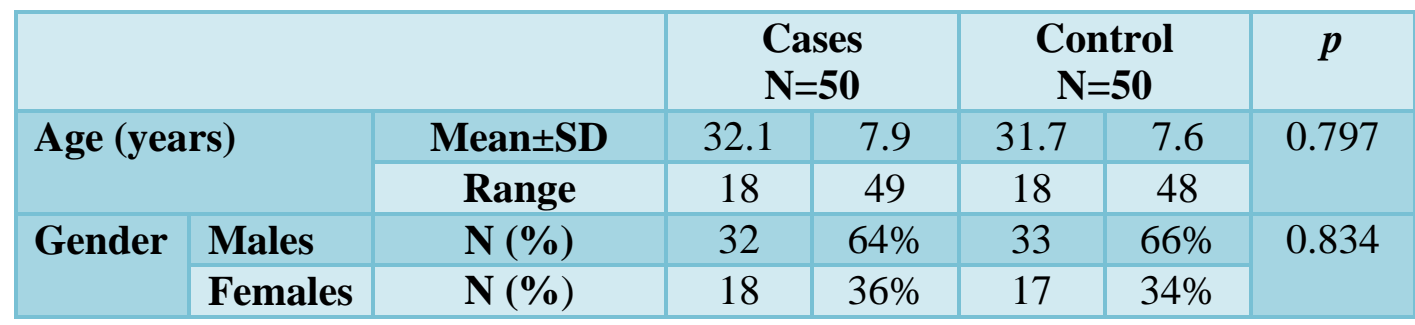

$\mathrm{SD}$, Standard deviation

Table.2 Collective phenotypic and genotypic results of studied cases and control

\begin{tabular}{|c|c|c|}
\hline Identification method & Case $\mathrm{N}=\mathbf{5 0}$ & Control $\mathrm{N}=\mathbf{5 0}$ \\
\hline KOH & Positive in $42(84 \%)$ & Positive in $5(10 \%)$ \\
\hline Culture & Positive in $39(78 \%)$ & Positive in $9(18 \%)$ \\
\hline Gram stain & Positive in $39(100 \%)$ & Positive in $9(100 \%)$ \\
\hline Urease & Positive in $39(100 \%)$ & Positive in $9(100 \%)$ \\
\hline Catalase & Positive in $39(100 \%)$ & Positive in $9(100 \%)$ \\
\hline Vitek2 & $\begin{array}{l}\text { 30(76.9\%) Malassezia furfur } \\
\text { 9(23.1\%) Unidentified }\end{array}$ & $\begin{array}{l}\text { 7(77.8\%) Malassezia furfur } \\
2(22.2 \%) \text { Unidentified }\end{array}$ \\
\hline MALDI-TOF & $\begin{array}{l}34(87.2 \%) \text { Malassezia furfur } \\
3 \text { (7.7\%) Malassezia globosa } \\
\text { 2(5.1\%) Unidentified }\end{array}$ & $\begin{array}{c}7(77.8 \%) \text { Malassezia furfur } \\
1 \text { (11.1\%) Malassezia globosa } \\
1(11.1 \%) \text { Unidentified }\end{array}$ \\
\hline Genomic PCR & $39(100 \%)$ Malassezia & $9(100 \%)$ Malassezia \\
\hline RFLP & $\begin{array}{l}\text { 35(89.7\%) Malassezia furfur } \\
\text { 4(10.3\%) Malassezia globosa }\end{array}$ & $\begin{array}{l}\text { 8(88.9\%) Malassezia furfur } \\
\text { 1(11.1\%) Malassezia globosa }\end{array}$ \\
\hline
\end{tabular}

Table.3 Comparison of different identification tests in positive cultures in both cases and controls

\begin{tabular}{|c|c|c|c|c|c|c|}
\hline \multicolumn{2}{|c|}{ Positive Culture } & \multicolumn{2}{|c|}{$\begin{array}{l}\text { Cases } \\
\mathrm{N}=39\end{array}$} & \multicolumn{2}{|c|}{$\begin{array}{c}\begin{array}{c}\text { Control } \\
\mathrm{N}=9\end{array} \\
\text { c }\end{array}$} & \multirow[t]{2}{*}{$p$} \\
\hline & & $\mathbf{N}$ & $\%$ & $\mathbf{N}$ & $\%$ & \\
\hline \multirow{2}{*}{ Vitek 2} & M. furfur & 30 & $76.9 \%$ & 7 & $77.8 \%$ & 0.956 \\
\hline & unidentified & 9 & $23.1 \%$ & 2 & $22.2 \%$ & 0.956 \\
\hline \multirow[t]{3}{*}{ MALDITOF } & M. furfur & 34 & $87.2 \%$ & 7 & $77.8 \%$ & 0.601 \\
\hline & M. globosa & 3 & $7.7 \%$ & 1 & $11.1 \%$ & 0.738 \\
\hline & unidentified & 2 & $5.1 \%$ & 1 & $11.1 \%$ & 0.472 \\
\hline \multirow[t]{2}{*}{ PCR-RFLP } & M. furfur & 35 & $89.7 \%$ & 8 & $88.9 \%$ & 0.940 \\
\hline & M. globosa & 4 & $10.3 \%$ & 1 & $11.1 \%$ & 0.940 \\
\hline
\end{tabular}


Table.4 Antifungal susceptibility of Malassezia isolated from patients and control

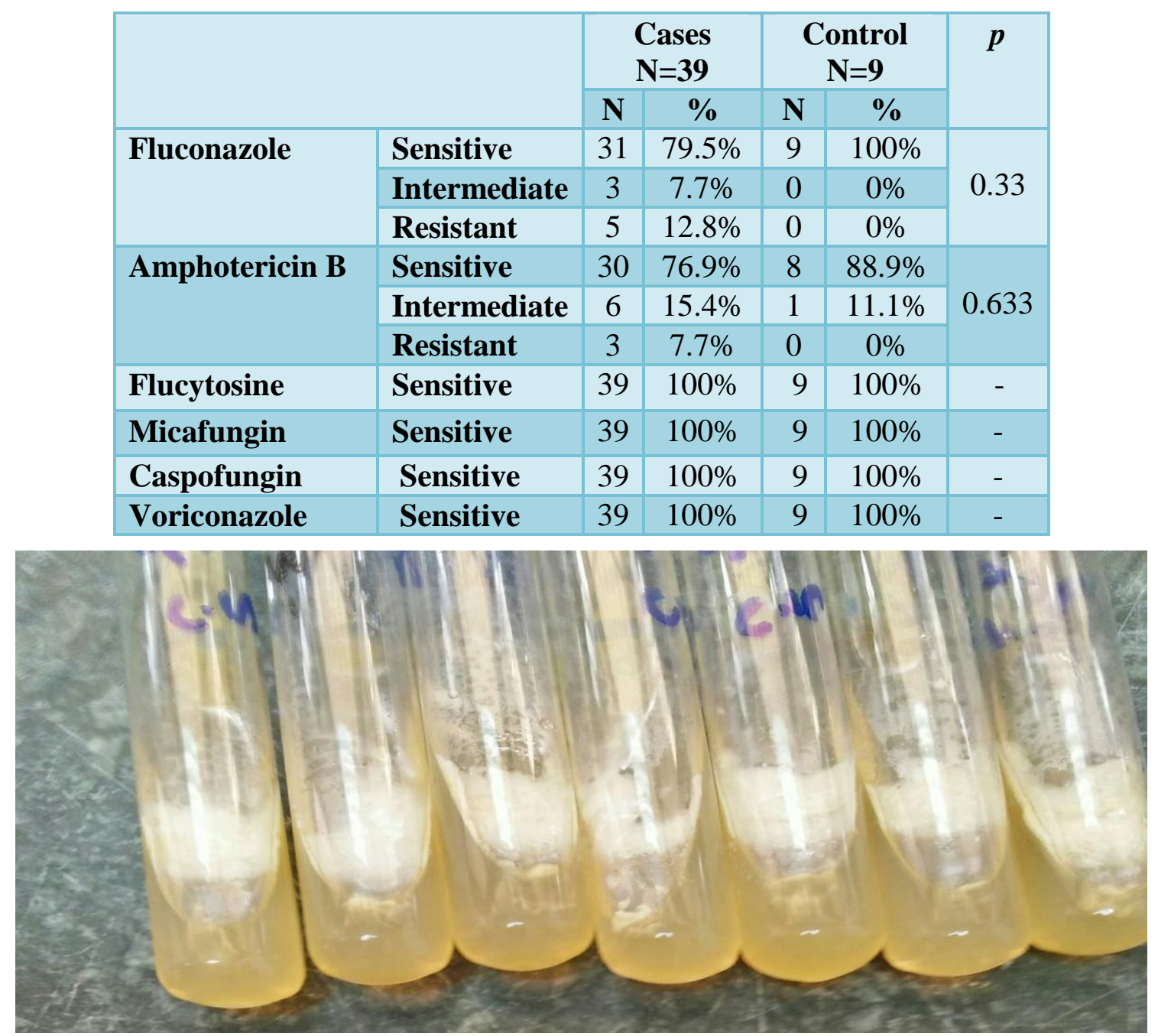

Figure.1 White and creamy colonies of Malassezia on SDA slantsoverlaid with olive oil

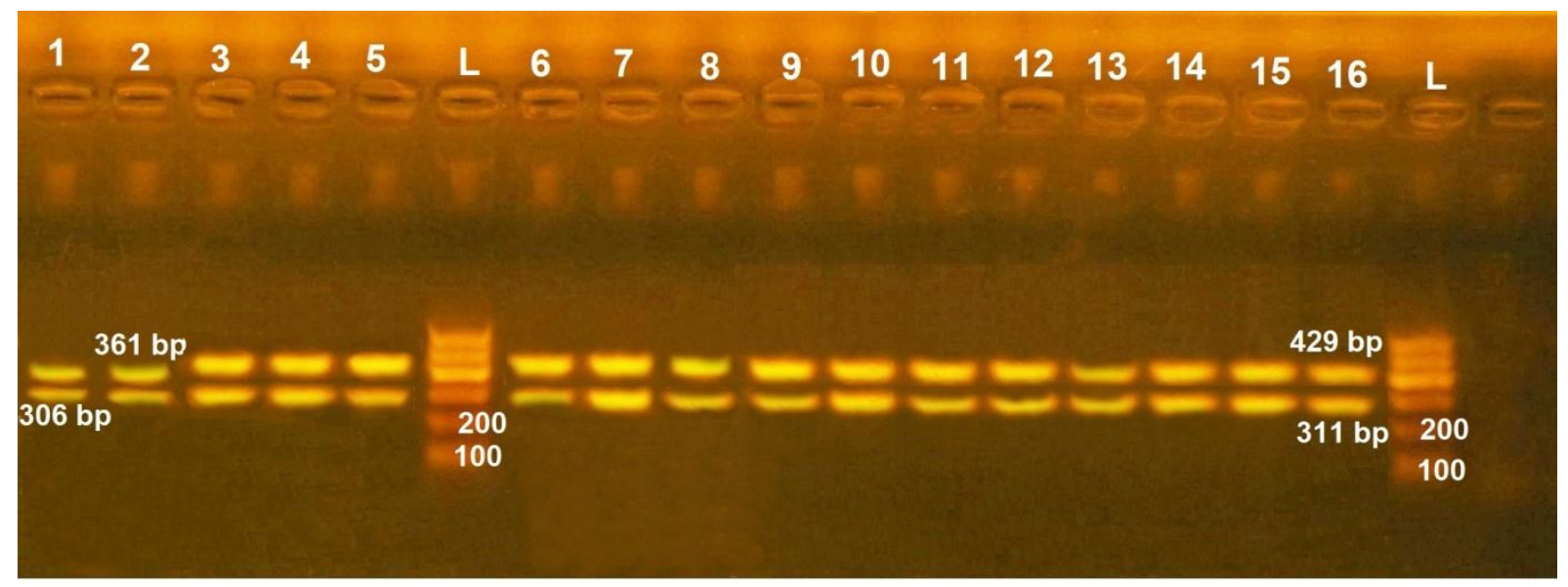

Figure.2 Two different patterns of bands by gel electrophoresis after restriction enzymes digestion; Band analysis showing Malassezia globosa (two bands, 306 and $361 \mathrm{bp}$ ) in first and second lane, Malassezia furfur (two bands, 311 and 429 bp) in the other lanes 
The results show that M.furfur and M.globosa are the most commonly encountered species in Egyptian patients with Pityriasis versicolor. PCR-RFLP method represents a considerably accurate technique in detection and identification of different Malassezia species, followed by MALDI-TOF and Vitek2.The most effective antifungal drugs against Malassezia were micafungin, caspofungin, voriconazole and flucytosine.

\section{References}

1. Theelen B, Cafarchia C, Gaitanis G, Bassukas ID, Boekhout T, Dawson Jr TL. Malassezia ecology, pathophysiology, and treatment. Medical mycology. 2018;56:S10-S25.

2. Awad AK, Al-Ezzy AIA, Jameel GH. Phenotypic Identification and Molecular Characterization of Malassezia Spp. Isolated from Pityriasis Versicolor Patients with Special Emphasis to Risk Factors in Diyala Province, Iraq. Open Access Macedonian Journal of Medical Sciences. 2019;7:707.

3. Harada K, Saito M, Sugita T, Tsuboi R. Malassezia species and their associated skin diseases. The Journal of dermatology. 2015;42:250-7.

4. Abdulrassol FA. The effect of the common determinants factors on the clinical aspects of pityriasis versicolor from Missan province. Misan Journal of Academic Studies. 2017; 16:22-33.

5. Saunte DM, Gaitanis G, Hay RJ. MalasseziaAssociated Skin Diseases, the Use of Diagnostics and Treatment. Frontiers in Cellular and Infection Microbiology. 2020;10:112-.

6. Mirhendi $\mathrm{H}$, Makimura $\mathrm{K}$, Zomorodian $\mathrm{K}$, Yamada T, Sugita T, Yamaguchi H. A simple PCR-RFLP method for identification and differentiation of 11 Malassezia species. Journal of microbiological methods. 2005;61:281-4.

7. Muhammad N, Kamal M, Islam $\mathrm{T}$, Islam N, Shafiquzzaman M. A study to evaluate the efficacy and safety of oral fluconazole in the treatment of tinea versicolor. Mymensingh medical journal: MMJ. 2009;18:31-5.

8. Ahmed SA, Roy CK, Jaigirdar QH, Khan RR,
Nigar I, Saleh AA. Identification of Malassezia species from suspected Pityriasis (versicolor) patients. Bangladesh Journal of Medical Microbiology. 2015;9:17-9.

9. Shah A, Koticha A, Ubale M, Wanjare S, Mehta P, Khopkar U. Identification and speciation of Malassezia in patients clinically suspected of having pityriasis versicolor. Indian journal of dermatology. 2013;58:239.

10. Moniri R, Nazeri M, Amiri S, Asghari B. Isolation and identification of Malassezia spp. in pytiriasis versicolor in Kashan, Iran. Pak J Med Sci. 2009;25:837-40.

11. Kabbin S, Vijaya D, Meundi M, Leelavathi B. A clinicomycological study of pityriasis versicolor with special reference to the calcofluor white stain. J Clin Diagn Res. 2011;5:1356-8.

12. Nakabayashi A, Sei Y, Guillot J. Identification of Malassezia species isolated from patients with seborrhoeic dermatitis, atopic dermatitis, pityriasis versicolor and normal subjects. Medical mycology. 2000;38:337-41.

13. Crespo Erchiga V, Ojeda Martos A, Vera Casano A, Crespo Erchiga A, Sanchez Fajardo F. Malassezia globosa as the causative agent of pityriasis versicolor. British journal of dermatology. 2000;143:799-803.

14. Gupta A, Bluhm R, Summerbell R. Pityriasis versicolor. Journal of the European Academy of Dermatology and Venereology. 2002;16:19-33.

15. Shoeib MA, Gaber MA, Labeeb AZ, ElKholy OA. Malassezia species isolated from lesional and nonlesional skin in patients with pityriasis versicolor. Menoufia Medical Journal. 2013;26:86.

16. Archana BR, Beena PM, Kumar S. Study of the distribution of malassezia species in patients with pityriasis versicolor in Kolar Region, Karnataka. Indian journal of dermatology. 2015;60:321.

17. Jang S-J, Lim S-H, Ko J-H, Oh B-H, Kim S$\mathrm{M}$, Song Y-C, et al., The investigation on the distribution of Malassezia yeasts on the normal Korean skin by $26 \mathrm{~S}$ rDNA PCRRFLP. Annals of dermatology. 2009;21:1826.

18. Chaudhary R, Singh S, Banerjee T, Tilak R. 
Prevalence of different Malassezia species in pityriasis versicolor in central India. Indian Journal of Dermatology, Venereology, and Leprology. 2010;76:159.

19. Shokohi T, Afshar P, Barzgar A. Distribution of Malassezia species in patients with pityriasis versicolor in Northern Iran. Indian journal of medical microbiology. 2009;27:321.

20. Denis J, Machouart M, Morio F, Sabou M, Kauffmann-LaCroix C, Contet-Audonneau N, et al., Performance of matrix-assisted laser desorption ionization-time of flight mass spectrometry for identifying clinical Malassezia isolates. Journal of clinical microbiology. 2017;55:90-6.

21. Lee YW, Lim SH, Ahn KJ. The application of 26S rDNA PCR-RFLP in the identification and classification of Malassezia yeast. Korean J Med Mycol. 2006;11:141.

22. Sharma A, Rabha D, Choraria S, Hazarika D, Ahmed G, Hazarika NK. Clinicomycological profile of pityriasis versicolor in Assam. Indian Journal of Pathology and Microbiology. 2016;59:159.

23. Li W, Zhang Z-W, Luo Y, Liang N, Pi X-X, Fan Y-M. Molecular epidemiology, in vitro susceptibility and exoenzyme screening of Malassezia clinical isolates. Journal of Medical Microbiology. 2020;69:436-42.

24. Gupta AK, Boekhout T, Theelen B, Summerbell R, Batra R. Identification and typing of Malassezia species by amplified fragment length polymorphism and sequence analyses of the internal transcribed spacer and large-subunit regions of ribosomal DNA.
Journal of clinical microbiology. 2004;42:4253-60.

25. Falk MHS, Linder MT, Johansson C, Bartosik J, Back O, Sarnhult T, et al., The prevalence of Malassezia yeasts in patients with atopic dermatitis, seborrhoeic dermatitis and healthy controls. Acta dermato-venereologica. 2005;85:17-23.

26. Gupta A, Kohli Y, Summerbell R, Faergemann J. Quantitative culture of Malassezia species from different body sites of individuals with or without dermatoses. Medical mycology. 2001;39:243-51.

27. Brunke S, Hube B. MfLIP1, a gene encoding an extracellular lipase of the lipid-dependent fungus Malassezia furfur. Microbiology. 2006;152:547-54.

28. Mayser P, Schäfer U, Krämer H-J, Irlinger B, Steglich W. Pityriacitrin-an ultravioletabsorbing indole alkaloid from the yeast Malassezia furfur. Archives of dermatological research. 2002;294:131-4.

29. Rojas FD, Sosa MdlA, Fernandez MS, Cattana ME, Cordoba SB, Giusiano GE. Antifungal susceptibility of Malassezia furfur, Malassezia sympodialis, and Malassezia globosa to azole drugs and amphotericin B evaluated using a broth microdilution method. Sabouraudia. 2014;52:641-6.

30. Hammer KA, Carson CF, Riley TV. In vitro activities of ketoconazole, econazole, miconazole, and Melaleuca alternifolia (tea tree) oil against Malassezia species. Antimicrobial agents and chemotherapy. 2000;44:467-9.

\section{How to cite this article:}

Randa EL-Sherbini, Noha Elmashad, Hanan Fathy, Mohammed Elshaer and Salah Agha. 2020. Molecular and Conventional Identification of Malassezia Species in Patients with Pityriasis Versicolor. Int.J.Curr.Microbiol.App.Sci. 9(06): 110-118. doi: https://doi.org/10.20546/ijcmas.2020.906.014 